\title{
Major impact of COVID-19 national containment on activities in the French northern comprehensive cancer center
}

\author{
Nicolas Penel ${ }^{1,2,3}$ (1) Ali Hammoudi ${ }^{1}$. Guillaume Marliot ${ }^{1}$. Antoine De Courreges ${ }^{2}$ - Malgorzata Cucchi ${ }^{1}$. \\ Xavier Mirabel ${ }^{1} \cdot$ Eric Leblanc $^{1}$ - Eric Lartigau ${ }^{1,2}$
}

Received: 18 November 2020 / Accepted: 22 January 2021 / Published online: 17 February 2021

○) Springer Science+Business Media, LLC, part of Springer Nature 2021

\begin{abstract}
The SARS-CoV-2 pandemic has significantly impacted cancer patient management. National and local recommendations to reduce SARS-CoV-2 transmission have been applied in a comprehensive cancer center located in Northern France. We prospectively measured key indicators for three successive eight-week periods: directly before, during, and right after the containment (from 16 March to 10 May 2020). Overall, the number of newly diagnosed and referred cancer patients in our hospital steadily increased $(1027 ; 1135$ and then $1704 ;+11 \%$ during containment and $+50 \%$ just after). To reduce patient transportation, teleconsultations were implemented. Teleconsulting activity steadily increased during the three periods $(5$, 2025 , and 2351). However, a marked decrease in the number of surgical procedures was observed $(448 ; 330$ and $288 ;-26 \%$ during containment and $-13 \%$ just after). We observed a slight decrease in the number of radiation therapy sessions (7761; 7328 and $7075 ;-6 \%$ during containment and $-3 \%$ just after) and in day-hospital cycles of IV systemic treatment (2891; 2736 and $2717 ;-5 \%$ during containment and $-1 \%$ just after). We observed an increase in the number of patients admitted to palliative care and a dramatic reduction in clinical trial enrollment. During this 24 -week period, organizational changes were mainly characterized by an increase in newly diagnosed cancer patient referral and the implementation of protective measures, such as teleconsultations. Activities in cancer surgery have decreased while radiotherapy and chemotherapy activities were stable.
\end{abstract}

Keywords Cancer patient management $\cdot$ SARS-CoV-2 epidemic $\cdot$ Teleconsultation $\cdot$ Care prioritization $\cdot$ Cancer surgery

\section{Introduction}

The novel human coronavirus named severe acute respiratory syndrome coronavirus 2 (SARS-CoV-2) emerged in Wuhan, China, in late 2019 and rapidly spread worldwide [1]. The SARS-CoV-2 epidemic started in the East and North of France in February 2020. To limit its transmission in the general population, different levels of preventive measures were set up (e.g., Emergency law dated March 23, 2020, National containment ...). Northern France was one of the most severely affected regions by the SARS-CoV-2

Nicolas Penel

n-penel@o-lambret.fr

Centre Oscar Lambret, Lille, France

2 Medical School, Lille University, Lille, France

3 Department of Medical Oncology, Centre Oscar Lambret, 3, rue Frederic Combemale, 59000 Lille, France pandemic (1100 people hospitalized and 1818 deaths between January and June 2020), especially during the national containment period (March 16, 2020 to June 2, 2020). During the pandemic, we had to reorganize the management of cancer patients, according to national and local authority recommendations and professional guidelines.

The Oscar Lambret Cancer center is the Comprehensive Cancer Center of Northern France (an area accounting four million inhabitants). This center manages 7800 cancer patients from all age groups yearly, with 3300 incident cases. The two most predominant malignancies were breast (1200 cases) and gynecological cancers (600 cases). This center provides a multidisciplinary approach including medical oncology, radiation oncology, surgery, supportive, and palliative cares. The National containment period was characterized by a drastic shift in patient care as hospital visits were limited to urgent matters only.

We had to limit the number of persons entering the cancer center in order to implement systematic temperature control 
and physical distancing. Services were modified according to the recommendations and available resources [2,3]. This required prioritizing curative-intent surgery and carefully balancing the risk-benefit ratio of each administered treatment. In the present study, we aimed to measure the impact of the national containment period on our hospital activity.

\section{Methods}

We prospectively measured different key indicators over three successive eight-week periods: (i) P1, from 20 January to $15 \mathrm{March}$, which reflected the activity with minimal impact from the COVID-19 pandemic; (ii) P2, from 16 March to 10 May 2020, which was the national containment period; and (iii) P3, from 11 May to 06 July, which was just after the containment period. Relative changes in activity have been expressed by three percentages: P2 versus $\mathrm{P} 1, \mathrm{P} 3$ versus $\mathrm{P} 2$, and $\mathrm{P} 3$ versus $\mathrm{P} 1$. Then, we analyzed 17 indicators during these three periods. "Patients" is the number of cancer patients who came at least once during the period. The "incident cases" is the number of newly diagnosed cancer patients in 2020, who came at least once during the period. "Outpatient visits" is the number of patients who came to the hospital for consultation, without administration of I.V. treatment, ambulatory surgery, or radiotherapy (including oral anticancer therapy). "Teleconsultation" is the number of consultations held through phone or Visio-conference. We collected the total number of cases discussed in the multidisciplinary board. We have collected the number of CT scan, MRI (magnetic resonance imaging), and pathology reports made during the three successive periods. We measured the number of I.V. "chemotherapy" cycles (this also includes targeted I.V. therapy such as trastuzumab and immune check-point inhibitor), drugs prepared for chemotherapy administration (e.g., carboplatin/etoposide administered for three days, required overall six drug units prepared par Pharmacy), and radiation therapy sessions. We measured the admissions to the medicine, surgery, and palliative care unit departments. Lastly, we collected the number of patients enrolled in phase I, phase II, and phase III cancer clinical trials. We also collected the number of enrolled patients under "COVID-19" studies (including a clinical trial in cancer patients with suspected COVID-19 and a large seroprevalence study in health cancer personals).

\section{Results}

The containment period was associated with a decrease in the overall number of patients coming to the hospital (Table 1). The number of treated incident cases steadily increased over the three successive periods. There was a significant decrease in outpatient visits during the containment period. In addition, just after the containment period, the outpatient visits did not reach the level observed at

Table 1 Activities during the three successive periods

\begin{tabular}{|c|c|c|c|c|c|c|}
\hline Items & $\begin{array}{l}\text { From } 20 \text { Jan to } \\
15 \operatorname{March}(\mathrm{P} 1)\end{array}$ & $\begin{array}{l}\text { From } 16 \text { March } \\
\text { to } 10 \text { May }(\mathrm{P} 2)\end{array}$ & $\begin{array}{l}\text { From } 11 \text { May } \\
\text { to } 06 \text { July (P3) }\end{array}$ & $\begin{array}{l}\text { Changes } \\
\text { P2 vs P1 }\end{array}$ & $\begin{array}{l}\text { Changes } \\
\text { P3 vs P2 }\end{array}$ & $\begin{array}{l}\text { Changes } \\
\text { P3 vs P1 }\end{array}$ \\
\hline Patients & 8248 & 5294 & 8472 & $-36 \%$ & $+60 \%$ & $+3 \%$ \\
\hline Patients aged to $\geq 65$ & 2994 & 1945 & 3157 & $-35 \%$ & $+62 \%$ & $+5 \%$ \\
\hline Incident cases & 1027 & 1135 & 1704 & $+11 \%$ & $+50 \%$ & $+66 \%$ \\
\hline Outpatient visits & 13,696 & 6507 & 10,741 & $-52 \%$ & $+65 \%$ & $-22 \%$ \\
\hline Teleconsultation & 5 & 2025 & 2351 & $+40,400 \%$ & $+16 \%$ & $+46,920 \%$ \\
\hline Cases discussed in multidisciplinary board & 1363 & 1063 & 980 & $-22 \%$ & $-8 \%$ & $-28 \%$ \\
\hline CT scans & 1732 & 1378 & 1458 & $-20 \%$ & $+6 \%$ & $-16 \%$ \\
\hline MRIs & 1119 & 699 & 963 & $-38 \%$ & $+38 \%$ & $-14 \%$ \\
\hline Pathology reports & 1559 & 1155 & 1055 & $-26 \%$ & $-9 \%$ & $-32 \%$ \\
\hline Delivered IV systemic treatment cycles in day hospital & 2891 & 2738 & 2717 & $-5 \%$ & $-1 \%$ & $-7 \%$ \\
\hline Drugs prepared for chemotherapy & 5673 & 5673 & 6010 & $+0 \%$ & $+6 \%$ & $+6 \%$ \\
\hline Radiation therapy sessions & 7761 & 7328 & 7075 & $-6 \%$ & $-3 \%$ & $-9 \%$ \\
\hline Admissions in surgery department & 448 & 330 & 288 & $-26 \%$ & $-13 \%$ & $-36 \%$ \\
\hline Admissions in medicine department & 441 & 371 & 404 & $-10 \%$ & $+9 \%$ & $-2 \%$ \\
\hline Admissions in palliative care unit & 36 & 49 & 47 & $+36 \%$ & $-4 \%$ & $+31 \%$ \\
\hline Enrollments in cancer clinical trials & 215 & 25 & 185 & $-88 \%$ & $+640 \%$ & $-14 \%$ \\
\hline Enrollment in COVID-19 studies & 0 & 0 & 330 & - & - & - \\
\hline
\end{tabular}


the beginning of the year. As recommended, the teleconsultation process was implemented during the containment period. Afterwards, patients and physicians still used this alternative for outpatient visits. We observed a steady decrease in the number of cases discussed at multidisciplinary tumor boards. The number of CT scans and MRIs decreased throughout the three successive periods and did not reach the baseline level just after containment. We observed a steady decrease in admissions in the surgical department and, consequently, pathology reports. At a lower extent, we also observed a steady decrease in the number of radiation therapy sessions. The number of delivered cycles of chemotherapy was stable across the successive periods. The number of molecules prepared by the Pharmacy Department slightly increased. The number of patients admitted to the palliative care unit sharply increased during the containment period and remained constantly above the baseline just after containment. Lastly, the impact of containment on clinical cancer research was very marked $(-89 \%)$, especially during containment. New studies focusing on COVID-19 have been rapidly initiated. Overall, we observed drastic changes in most activities. We can classify the activities into three patterns: (i) steadily increasing activities (incident cases, teleconsultations and drugs prepared for chemotherapy, admissions in palliative care unit); (ii) steadily decreasing activities (admissions in surgery department, pathology reports, delivered cycles of chemotherapy and cases discussed in multidisciplinary tumor boards); and (iii) activities with a drastic decrease during containment, followed by a subsequent increase that did not reach the baseline level (imaging, admissions in medicine department, radiation therapy sessions, enrollments in cancer clinical trials).

\section{Discussion}

The observed changes in activities were more rapid and drastic than expected. There were three different patterns of activities, requiring flexible resource management.

This study had some limitations. The indicators described the activities but their impact on patient outcome was not estimated. For example, we did not document the shift from I.V. systemic to oral anticancer treatment. The duration of this study was relatively short, and the SARS$\mathrm{CoV}-2$ epidemic is still ongoing, with further national and local recommendations still pending (possible new containment). We did not describe patient management, but we collected activity indicators at the hospital level. We are planning a more precise analysis of patient care for a homogeneous patient group (e.g., localized breast cancer).
However, the observed changes are impressive and warrant discussion.

Specific activities steadily increased during the three successive periods. We observed a constant increase in incident cases treated in the Comprehensive Cancer Center $(+66 \%)$. Local hospitals in the area were the first to be mobilized for the management of SARS-CoV-2 the epidemic. As a result, many incident cases were referred to the Comprehensive Cancer Center for immediate management. To avoid transportation and maintain physical distancing within the hospital, teleconsultations (by phone or video) and tumor boards have been implemented. Until the SARS-CoV-2 epidemic, a limited number of teleconsultations have been reimbursed by the French Social Security. In emergency situations, the French Social Security provided guidelines and authorization for implementing teleconsultations. Lastly, the number of patients admitted to the palliative care unit has markedly increased. This suggested that in the context of SARS-CoV-2 epidemic, the risk-benefit ratio of maintaining or starting treatment was more frequently unfavorable. Furthermore, some local hospitals closed their palliative units and converted them to COVID-19 patient management facilities. During COVID-19, our hospital as a regional comprehensive center faced a sudden and major increase in referred incident cancer patients.

Some activities constantly decreased during the two successive periods. The most impressive change was the decrease in admission for surgical procedures $(-36 \%)$, resulting in the decrease in pathology reports $(-32 \%)$ and multidisciplinary tumor board discussions $(-28 \%)$.

This marked drop in surgical activities may be due to two factors. First, during containment there was a dramatic decrease in breast, colorectal $[4,5]$, and cervical cancer screening. At the same time, we faced anesthetic drug shortages (such as midazolam, propofol, curares) due to increased use for managing COVID-19 patients in intensive care units and decreased production, caused by COVID-19 as well. Consequently, a national contingency plan was applied to address the shortages. The number of surgical procedures, especially those for non-cancer cases, decreased during this period. Surgeons and anesthesiologists had to prioritize curative-intent surgeries, followed by palliative surgeries. All other reconstructive and/or non-urgent procedures were postponed (Appendix). They reorganized the operative theaters, hospitalization wards, and operative programs. Anesthetic protocols and surgical techniques were modified to maintain quality patient care despite drug shortages while limiting the risk of in-hospital contamination. Regular virtual meetings with the direction and other medical poles or departments (oncology, 
imaging, pathology, anesthesiology, radiation therapy, local pharmacy) were organized to adjust the local management, according to the pandemic situation (containment then progressive un-containment). These adjustments considered national and regional information, specific recommendations from scientific societies, and pharmacy state of stocks.

Due to the pandemic, compliance with the cancer screening recommendation was poor and was not a shortterm priority. Furthermore, screening programs were disorganized during this period. The cancer screenings in September 2020 remained limited, compared to the number of new non-palpable breast and small colon cancers in the previous year. We do not see as many new cases of non-palpable breast cancers or small colon cancers as in 2019. However, symptomatic tumors were still diagnosed. These patients were referred to the comprehensive center for multidisciplinary management. Further studies may evaluate the impact of decreased cancer screening on survival.

The department of medicine oversees inpatient administration for chemotherapy, supportive or palliative care, diagnostic procedures, and management of complications or comorbidities requiring hospital stays. We observed a transient and limited decrease in admission. During the study, less than 30 patients were suspected to suffer from COVID-19. Suspected cases were referred to the local hospital. We established units dedicated to COVID-19 patient management during the containment period and then reassigned this to surgery just after containment. The number of I.V. systemic treatment cycles slightly decreased during the study period $(-7 \%)$, but these treatments were more complex, including polychemotherapy. Treated patients were selected, and curative-intent (including adjuvant or neoadjuvant) regimens were prioritized. The risks and benefits of chemotherapy must be carefully balanced in this context. Advanced malignancy is fatal, and the COVID-19 mortality in solid cancer patients is about 25\%. COVID-19 increased the mortality rate in cancer patients by three times $[6,7]$. Protecting cancer patients receiving treatment from COVID19 is challenging.

Hypofractionated radiation therapy was applied when possible to limit patient trips [8]. This caused a minor decrease in the number of radiotherapy sessions.

Clinical research activity drastically changed during this 24-week period. Enrolling new patients and maintaining participation in clinical trials was challenging during this pandemic period [9]. Clinical trial enrollment is an irreplaceable alternative in patients who have exhausted their approved treatment options. One of the major roles of the comprehensive cancer center is innovating therapeutic strategies for patients. However, prioritizing patient safety and data quality are main concerns for investigators, sponsors, and authorities [10]. Conducting cancer trials in the context of the pandemic is challenging. We observed a sharp decrease in cancer clinical trial enrollment and a shift to trials investigating COVID-19 (e.g., seroprevalence study in health workers, investigational new treatment in cancer patient with suspected COVID-19, psychological impact of teleconsultation in cancer patients ...) in our center.

To conclude, our comprehensive cancer center faced a sudden and major increase in newly diagnosed cancer patient referrals. However, there was a decrease in administered treatment and procedures (e.g., $-36 \%$ of surgical procedures, $-9 \%$ of radiation therapy sessions, and $-7 \%$ of IV systemic treatment administration), suggesting a drastic selection for treatment administration. The decrease was more evident during the containment period. Beyond resource reallocation and flexibility in care organization, this documents complex clinical and managerial situations and ethical dilemmas [11].

\section{Appendix}

National and local measures.

National protective measures (from 16 March)

Impose mandatory home confinement from March 16 to June 11, 2020

Promote hand washing, mask, and physical distancing

Close all schools and all universities

Shutdown of non-essential businesses and other venues open to the public

Ban gatherings of more than 100 people

Limit use of public transport for going to work if presence at the workplace is essential

Ban on all travel except relating to professional activity, buying essential goods, health or family reasons, or brief individual exercise. Those outside the home were required to carry identification and a signed and dated declaration for any travel

All suspected or confirmed cases of death due to COVID-19 were to be placed in coffins immediately

National organization of cares during SARS-CoV-2 pandemic Avoid non-essential health care

Implement hand washing, mask, and physical distancing within hospital

Identify units for management of suspected or proven COVID-19, and declare cases to authorities

Tertiary hospitals had prioritized management of patients with suspicion or confirmed cases of COVID-19

In every hospital, set up units dedicated to COVID-19 patient management 
Within Oscar Lambret Cancer center (from February 20, 2020)

Set up a hospital-wide crisis team responsible for coordinating measures between departments

Promote hand washing, mask, and physical distancing for all staff members

In-house production of hydroalcoholic solution: provide and distribute masks to health care providers

Provide mask for outpatients and visitors

Instruct patients not to visit the hospital if they have symptoms indicative of possible COVID-19 (unless urgent attention is required) Screening of patients accessing to the hospital: Identify potential COVID-19 cases before hospital entry (phone call before hospitalization), at hospital entry (nurses systematically see every patient or visitor before admission: temperature check and systematic questionnaire about COVID-19-related symptoms), and in the event of COVID-19-related symptoms, test the patient as soon as possible During containment period, set up a dedicated unit for management of suspected or confirmed COVID-19 cases with dedicated staff (nurses and physicians). Quickly isolate patients with COVID-19 in this dedicated unit, with the intent of referring, if possible, these patients to regional collaborating in charge of management of COVID-19 patients

Reduce the number of workers within the hospital: promote telework, close the staff canteen

Avoid staff gathering (e.g., virtual multidisciplinary team meetings)

Reduce the presence of non-essential people: if possible, limit family visits, paramedic stay outside the hospital ...

Reduce or postpone non-essential care (e.g., breast reconstruction), promote teleconsultations, reduce the number of hospital stays (e.g., Use of hypofractionated radiation therapy for patients requiring palliation; use of oral chemotherapy rather than I.V. chemotherapy, if possible, avoid chemotherapy with high risk of febrile neutropenia; postpone non-essential radiological assessments ...)

Prioritize curative-intent cares

Acknowledgements We are grateful to Séverine Marchant for her help in manuscript editing.

Author contributions NP: Conceptualization, methodology, formal analysis, writing - original draft, writing - review and editing. $\mathrm{AH}$, GM, AC, MC, XM, and EL: Investigation, writing — review and editing. EL: writing - review and editing, supervision, funding acquisition.

Funding This research received no specific grant from any funding agency in the public, commercial, or not-for-profit sectors.

\section{Compliance with ethical standards}

Conflict of interest The authors declare that there are no competing interests.

\section{References}

1. Hui DS, Azhar E, Madani TA, Ntoumi F, Kock R, Dar O, et al. The continuing 2019-nCoV epidemic threat of novel coronaviruses to global health - the latest 2019 novel coronavirus outbreak in Wuhan China. Int J Infect Dis. 2020;91:264-6.

2. Onesti CE, Rugo HS, Generali D, Peeters M, Zaman K, Wildiers $\mathrm{H}$, et al. Oncological care organisation during COVID-19 outbreak. ESMO Open. 2020;5:e000853.

3. Jazieh AR, Chan SL, Curigliano G, Dickson N, Eaton V, GarciaFoncillas J, et al. Delivering cancer care during the COVID-19 pandemic: recommendations and lessons learned from ASCO global webinars. JCO Glob Oncol. 2020;6:1461-71.

4. Belle A, Barret M, Bernardini D, Tarrerias AL, Bories E, Costil V, et al. Impact of the COVID-19 pandemic on gastrointestinal endoscopy activity in France. Endoscopy. 2020. https://doi. org/10.1055/a-1201-9618.

5. Del Vecchio BG, Calabrese E, Biancone L, Monteleone G, Paoluzi OA. The impact of COVID-19 pandemic in the colorectal cancer prevention. Int J Colorectal Dis. 2020;35:1951-4.

6. Cattaneo C, Daffini R, Pagani C, Salvetti M, Mancini V, Borlenghi $\mathrm{E}$, et al. Clinical characteristics and risk factors for mortality in hematologic patients affected by COVID-19. Cancer. 2020;126:5069-76.

7. Fillmore NR, La J, Szalat RE, Tuck DP, Nguyen V, Yildirim C, et al. Prevalence and outcome of COVID-19 infection in cancer patients: a national veterans affairs study. J Natl Cancer Inst. 2020. https://doi.org/10.1093/jnci/djaa159.

8. Caravatta L, Rosa C, Di Sciascio MB, Tavella Scaringi A, Di Pilla A, Ursini LA, et al. COVID-19 and radiation oncology: the experience of a two-phase plan within a single institution in central Italy. Radiat Oncol. 2020;15:226.

9. U. S. Food and Drug Administration 2020 FDA guidance on conduct of clinical trials of medical products during COVID-19 public health emergency, Guid Doc, pp. $1-29$ https://www.fda. gov/regulatory-information/search-fda-guidance-documents/fdaguidance-conduct-clinical-trials-medical-products-during-covid -19-public-health-emergency.

10. Arai RJ, Moniz CMV, Chen ATC, Mak MP, Chammas R, et al. Managing oncology clinical trials during COVID-19 pandemic. Contemp Clin Trials Commun. 2020;19:100637.

11. Blot F, Dumont SN, Vigouret-Viant L, Verotte N, Rossignol J, Rieutord A, et al. Ethical issues related to the COVID-19 pandemic in patients with cancer: experience and organisations in a French comprehensive cancer centre. BMJ Support Palliat Care. 2020. https://doi.org/10.1136/bmjspcare-2020-002504.

Publisher's Note Springer Nature remains neutral with regard to jurisdictional claims in published maps and institutional affiliations. 